\title{
Cellular traction forces increase during consecutive mechanical stretching following traction force attenuation
}

\author{
Akira TSUKAMOTO ${ }^{* * * *}$, Katie R. RYAN*, Yusuke MITSUOKA*, Katsuko S. FURUKAWA ${ }^{* *}$ \\ and Takashi USHIDA* \\ *Department of Mechanical Engineering, The University of Tokyo, 7-3-1 Hongo, Bunkyo-ku, Tokyo, Japan \\ E-mail: ushida@mech.t.u-tokyo.ac.jp \\ **Department of Biomedical Engineering, The University of Tokyo, 7-3-1 Hongo, Bunkyo-ku, Tokyo, Japan \\ ${ }^{* * *}$ Current affiliation: Department of Applied Physics, National Defense Academy of Japan, \\ 1-10-20 Hashirimizu, Yokosuka-shi, Kanagawa, Japan
}

Received: 22 February 2017; Accepted: 24 February 2017

\begin{abstract}
Adherent cells generate traction forces, which are generated by and transmitted along the actin cytoskeleton to the underlying external substrate via focal adhesions. These cell generated forces are fundamental for maintaining cell shape and driving cell migration as well as triggering signaling pathways to promote processes such as differentiation and proliferation. Here we investigate how mechanical stretch affects traction forces. Following moderate mechanical stretching and release, traction forces are increased and then return to basal levels. However, when cells are stretched with relatively large strains, $>10 \%$, traction forces fail to return to basal levels and are attenuated. In this study, we investigate whether cells experiencing attenuated traction forces, following a large strain, can still respond to subsequent mechanical stretching. Traction forces were measured in cells following a consecutive $12 \%$ increase in mechanical stretch. We show that, even after traction force attenuation occurs, cells are able to respond to consecutive mechanical stretching by increasing traction force. Visualization of stress fibers, with GFP-actin, indicates that the attenuation of traction force is accompanied by a decrease in stress fiber integrity during mechanical stretching. The ability of cells to increase traction force during a second round of stretching, following attenuation, maybe generated by intact stress fibers that escape damage.
\end{abstract}

Key words: Traction force, Mechanical stretch, Actin stress fibers, HUVEC

\section{Introduction}

Traction forces are contractile forces that are carried along the actin cytoskeleton of adherent cells enabling cell migration (Riveline et al., 2001). Generated by actomyosin contractility, traction forces are transmitted via actin stress fibers to focal adhesions and the extracellular matrix (Burton et al., 1999; Gavara et al., 2008; Wang and Lin, 2007). Cells use traction forces for migration, cell shape maintenance, to probe their surrounding environment and to generate mechanical signals (Pelham and Wang, 1997). Understanding how traction forces are regulated is crucial for the understanding of fundamental processes such as wound healing, angiogenesis and metastasis. When cells are subjected to mechanical stretching traction forces are increased (Gavara et al., 2008; Lam et al., 2012; Munevar et al., 2004; Nagayama et al., 2011). Once cells are released from the mechanical stretching strain, traction forces return to basal levels. This return depends on the magnitude of strain being imposed on the cell during mechanical stretching. When the strain is relatively small, such as $5.5 \%$, traction forces return to basal levels after release. In contrast, when the strain is relatively large, such as $11 \%$, traction forces fail to return to basal levels but diminish to lower values (Gavara et al., 2008). This attenuation of traction forces is also observed following repeated stretching when the strain is $10 \%$ 
(Krishnan et al., 2012). This means that cells are subjected to repeated rounds of mechanical stretching under conditions when traction forces are attenuated.

Alterations in the actin cytoskeleton have been reported to play a role in the attenuation of traction forces. Rupturing and unfolding of both actin filament cross-links and actomyosin cross-bridges have been reported, as well as changes in actin polymerization following large strain mechanical stretching (Costa et al., 2002; Gavara et al., 2008; Lim et al., 2012; Rosenblatt et al., 2007; Trepat et al., 2007; Trepat et al., 2006). Increases in traction force depend on an intact actin cytoskeleton (Gavara et al., 2008; Lam et al., 2012; Munevar et al., 2004; Nagayama et al., 2011). This disruption of the actin cytoskeleton, following large strain mechanical stretching, may cause the attenuation of traction force.

In this study, we investigated whether traction forces are able to increase once attenuation has occurred following repeated mechanical stretching with strains of $12 \%$. We utilized traction force microscopy to measure traction forces, which were calculated from the displacement of cellular substrates (Wang and Lin, 2007). We also analyzed actin stress fiber dynamics in situ by visualizing actin stress fibers with GFP-actin. Here we show that following mechanical stretching with large strains traction force attenuation occurs accompanied by the disruption of actin stress fibers. However, following another round of mechanical stretching cells were still able to significantly increase traction force indicating the cell still retains the ability to increase traction force following attenuation.

\section{Materials and Methods}

\subsection{Stretch chambers}

PDMS (RTV4168, Dow Corning Toray, Japan) was mixed at a ratio of 10:1 (base to curing agent), degassed and poured into a custom made mold and incubated at $60^{\circ} \mathrm{C}$ for 2 hours to solidify and adhere to stainless steel grippers (Fig. 1A). Prior to cell seeding, the stretch chamber $(20 \mathrm{~mm} \times 30 \mathrm{~mm}$ in area and $100 \mu \mathrm{m}$ in thickness in the center of the PDMF form) was treated as follows (Fig 1B)... 1. Coated with gelatin as previously described (Wang and Lin, 2007) with modification, in brief, acid-processed beef bone gelatin (Nitta Gelatin, Japan) was dissolved in distilled water $(10 \%(\mathrm{w} / \mathrm{v}))\left(60^{\circ} \mathrm{C}\right.$ for 1 hour $)$. The gelatin solution was neutralized with $\mathrm{NaOH}(1 \mathrm{M})$, filter sterilized, maintained at $60^{\circ} \mathrm{C}$ and transglutaminase $(0.3 \%(\mathrm{w} / \mathrm{v})$ Ajinomoto, Japan) was added. The gelatin solution was then applied to the surface of the stretch chamber, which was under a pretension ( $2 \mathrm{~mm}$ of displacement) to prevent sagging and incubated at $37^{\circ} \mathrm{C}$ overnight allowing the gelatin solution to cross-link and set into a gel $\sim 1 \mathrm{~mm}$ thick. 2 . Fluorescence microbeads $(\phi 0.2 \mu \mathrm{m}, \mathrm{F} 8810$, Invitrogen, USA) diluted in distilled water $(10 \%(\mathrm{v} / \mathrm{v}))$ were applied to the surface of the cross-linked gelatin gel, incubated for $1 \mathrm{~min}$, and the surface was washed three times with distilled water. To stabilize the attachment of microbeads to the gelatin the surface was air-dried for 60 min. 3. The gelatin gel was then coated with collagen by incubating it with collagen solution $\left(0.05 \mathrm{mg} / \mathrm{ml}\right.$ in PBS, Koken, Japan) at $4^{\circ} \mathrm{C}$ for 8 hours.

The Young's modulus of the gelatin gel coating the PDMS stretch chamber was measured using stainless steel spheres (1 mm in radius, Funabe Seiko, Japan). Using Hertzian contact stress theory, the Young's modulus was determined from the depth of the indentation under the sphere (Gaudet et al., 2003; Teixeira et al., 2009). The Young's modulus of the gel was $11.7 \pm 0.6 \mathrm{kPa}(\mathrm{n}=12)$. Pavan et al have shown that the elasticity of gelatin gels are not significantly effected by the strain loads which were used in this study, i.e., 12\% (Pavan et al., 2010). Thus, the Young's modulus of the gelatin gel under strain of $12 \%$ was estimated to be equal to the Young's modulus under static condition.

\subsection{Cell culture}

Human umbilical vein endothelial cells (HUVECs; Sanko Junyaku, Japan) were cultured with endothelial cell medium (Sanko Junyaku) at $37^{\circ} \mathrm{C}, 5 \% \mathrm{CO}_{2}$. The passage number of HUVECs was limited to 3 - 8. HUVECs were transfected with GFP-actin (Clontech, USA) using Lipofectamine 2000 (Invitrogen) according to the manufacturer's instructions. Following transfection, HUVECs were reseeded onto treated stretch chambers (Fig. 1) at a density of $1 \times 10^{4}$ cells $/ \mathrm{cm}^{2}$ and incubated for $1-2$ days prior to testing.

\subsection{Mechanical stretching of cells}

Cells were washed with HEPES buffered saline solution (HBSS: (in mM): $\mathrm{NaCl} 130, \mathrm{KCl} 5.4, \mathrm{CaCl}_{2} 1.8, \mathrm{MgCl}_{2}$ 0.8 , glucose 5.5, HEPES 20; $\mathrm{pH}$ adjusted to 7.4 with $\mathrm{NaOH}$ ) and all experiments were performed using HBSS. To load 
cells with mechanical stretch, the stretch chambers were fixed to a custom-made stretch loader (Mitsuoka et al., 2012). Equi-uniaxial stretch, with a strain of $12 \%$ and a strain speed of $12 \% / \mathrm{sec}$, were loaded onto the cells. The temperature around the stretch chamber was maintained at $37^{\circ} \mathrm{C}$.

A

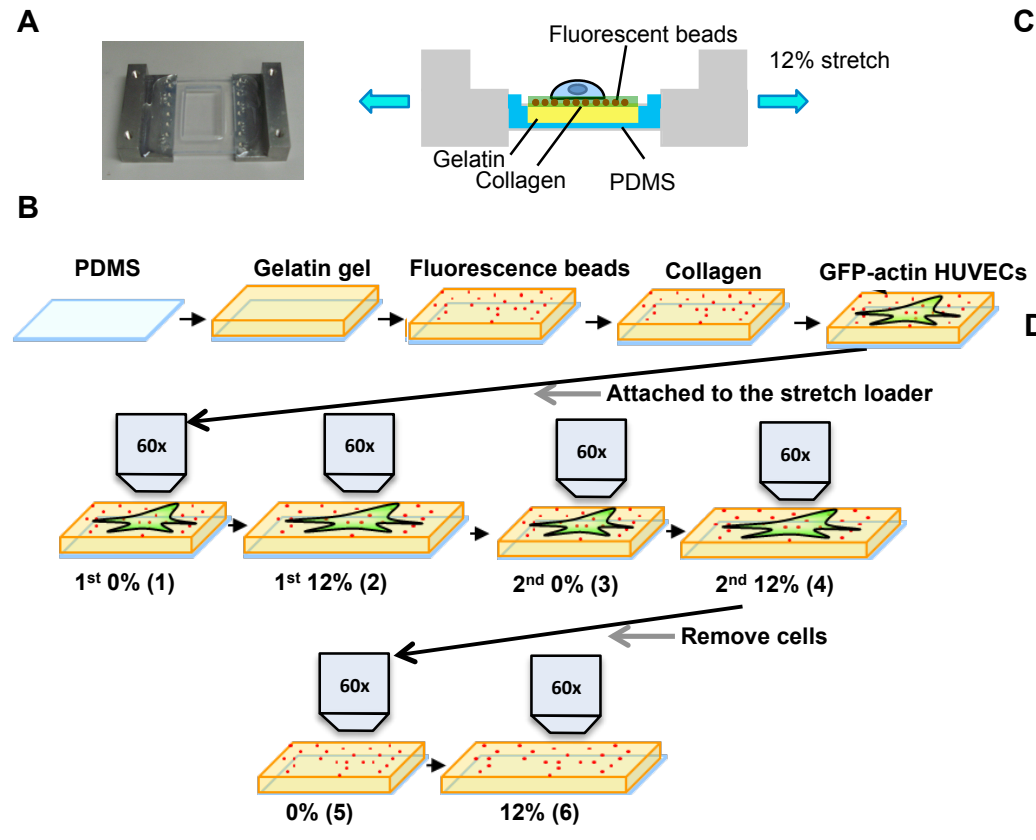

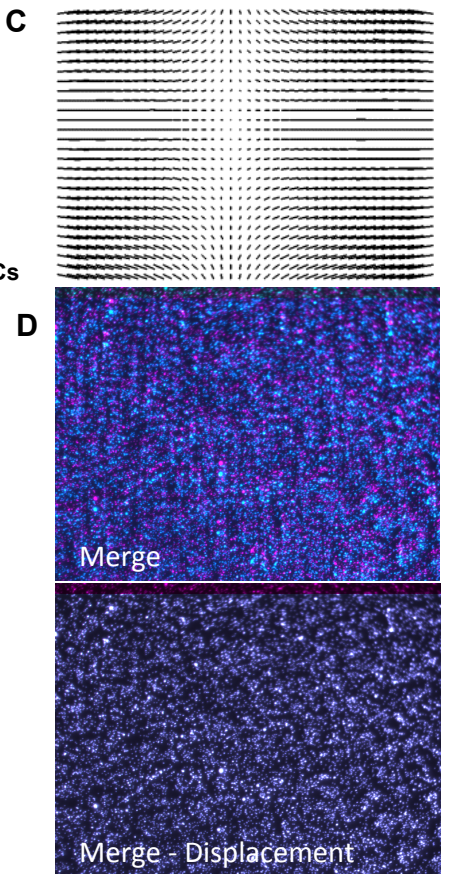

Fig. 1. Stretch chamber and protocol for sample preparation and traction force measurements in cells undergoing consecutive mechanical stretching. (A) Stretch chambers were molded from PDMS to stainless steel grippers. (B) The thin PDMS film of the stretch chamber was coated with gelatin gel, fluorescent beads (enabling traction force measurements) and collagen before being seeded with HUVECs transfected with GFP-actin. Fluorescence images were obtained at six different points (1-6) for each sample, GFP-actin and fluorescent beads (1-4) and fluorescent beads only (5-6). Following the second $12 \%$ stretch, cells were detached from the gel with air bubbles. $0 \%$ stretch refers to static conditions without mechanical stretching. (C) Displacement of fluorescence beads on gelatin gel under $12 \%$ stretch was analyzed with PIV analysis. (D) After one cycle of stretch, the fluorescence beads on gelatin gel returned to their former positions after image displacement was corrected for (Red: before stretch, Blue: after stretch, Merge: before image displacement correction, Merge - Displacement: after image displacement correction).

\subsection{Traction force measurement}

Images of the fluorescent beads on the surface of the gel were captured using an epifluorescence microscope (BX51, Olympus, Japan), with an excitation wavelength of $580 \mathrm{~nm}$ and emission wavelength of $605 \mathrm{~nm}$. The microscope was equipt with a 60x water-immersion objective lens ( $\mathrm{NA}=1.1$, Olympus), a multi-band fluorescence filter (Semrock, USA) and a 3CCD camera (Hamamatsu Photonics, Japan). For traction force microscopy, images of fluorescent beads are required to be obtained in the presence and absence of cells. In this study, cells were detached using air bubbles generated by a microinjector (Eppendorf, Germany). The air bubbles induce high shear stresses, which results in cells detaching from the gelatin gel (Decave et al., 2002).

Particle image velocimetry (PIV), custom-written JAVA software, was used to calculate substrate displacement (Butler et al., 2002; Tolic-Norrelykke et al., 2002). For PIV, the extracted fluorescence images were 420 pixels $\times 630$ pixels, which corresponded to $90 \mu \mathrm{m} \times 135 \mu \mathrm{m}$. With PIV, displacements were obtained every 14 pixels $\times 14$ pixels, which corresponded to $3 \mu \mathrm{m} \times 3 \mu \mathrm{m}$. From the displacement fields, the traction forces were calculated using 3D finite element method (FEM; Ansys, USA) (Yang et al., 2006). The Poisson ratio was set to 0.4 (Markidou et al., 2005). The analysis volume for 3D FEM was set to $90 \mu \mathrm{m} \times 135 \mu \mathrm{m} \times 500 \mu \mathrm{m}$. Meshes were created either every $3 \mu \mathrm{m} \times 3 \mu \mathrm{m} \times 4$ $\mu \mathrm{m}$ in the analysis volume within $20 \mu \mathrm{m}$ from cell-attachment regions, or every $3 \mu \mathrm{m} \times 3 \mu \mathrm{m} \times 30 \mu \mathrm{m}$. The boundary conditions, i.e., displacements, required for 3D FEM were defined as follows (Tolic-Norrelykke et al., 2002): In cell-attachment regions, displacements were defined by PIV calculations. The cell-attachment regions were identified 
using fluorescence images of GFP-actin, obtained as described in section 2.5. In regions without cell attachment, displacements were defined as zero. After 3D FEM, traction forces were obtained with the root mean square (RMS) of force vectors, which were perpendicular to the surface of the cell-attachment regions. Finally, traction forces in the cell-attachment regions were averaged then multiplied by the area of the cell-attachment region and defined as traction forces exerted by single cells.

PIV analysis confirmed that the $12 \%$ stretch had no significant effect on the displacement of the fluorescence beads on gelatin gel. There was however a shift in before and after image of the beads following stretch, this was due to the manual reacquisition of the cell following stretch, which results in a parallel displacement of the bead images (Fig. 1D). After correcting for the parallel shift in the image, displacement of fluorescence beads after one cycle stretch was 0.31 \pm 0.06 pixel $(\mathrm{N}=12)$, which was not significantly different from the non-stretched samples $0.31 \pm 0.11$ pixel $(\mathrm{N}=9)$.

\subsection{Stress fiber imaging}

Stress fibers, labeled with GFP-actin, were imaged in parallel with the fluorescent beads and captured with the 3CCD camera, using an excitation wavelength of $480 \mathrm{~nm}$ and an emission wavelength of $520 \mathrm{~nm}$. Images were enhanced using the FFT bandpass filter function, sharpen function and brightness/contrast function in ImageJ (NIH, USA). Fluorescence intensities along stress fibers were quantified using the analysis function in ImageJ after setting analysis lines on stress fibers.

\subsection{Experimental procedure}

The stretch chamber was attached to a custom-made stretch loader and the following protocol was carried out (Fig. 1B): Images of the fluorescent beads and stress fibers were captured under static condition, i.e. $0 \%$ stretch. $12 \%$ mechanical stretch was applied to the stretch chamber. Cell location was altered following stretching so cells were reacquired and images of the fluorescent beads and stress fibers were captured under mechanical stretching condition, i.e. $12 \%$ stretch, after 1 min of stretch. Mechanical stretching was then released returning the stretch chamber to $0 \%$ stretch. Cells were left for $30 \mathrm{~min}$, to allow time for an equilibrium to be reached following the perturbations in the cytoskeleton (Krishnan et al., 2009), before capturing images of the fluorescent beads and stress fibers at the second $0 \%$ stretch. The stretch chamber was then loaded with the second round of mechanical stretching, $12 \%$ stretch, cells were reacquired and images were captured. After the relaxation of the stretch chamber, the third $0 \%$ stretch, cells were detached with air bubbles as described in section 2.4. Images of the fluorescent beads, in the absence of cells, were captured at both $0 \%$ and $12 \%$ stretch.

\subsection{Statistical analysis}

Data are expressed as means \pm SEMs. Differences between groups were calculated by paired Student's T-test. Statistical significance is indicated by "*" when the $\mathrm{p}$-value $=<0.05$, and $" * * "$ when $\mathrm{p}$-value $=<0.01$.

\section{Results}

\subsection{Traction force increases following attenuation during mechanical stretch}

Traction force microscopy was used to measure the traction forces exerted by HUVECs on gelatin gels (Fig. 1) in static conditions $(0 \%$ stretch, $=335 \mathrm{nN})$ (Fig 2). This value was consistent with traction forces reported by previous studies (Gavara et al., 2006; Lam et al., 2012; Nagayama et al., 2011). For each stage of the consecutive mechanical stretching and release cycle: the first $0 \%$ stretch, first $12 \%$ stretch, second $0 \%$ stretch and second $12 \%$ stretch, traction forces were recorded for each cell (Fig. 2A). A profile of the traction forces produced by cells was generated over the consecutive mechanical stretching and release cycle (Fig. 2B). During the first $12 \%$ stretch there was a slight but significant increase in traction force compared to the baseline traction force established in the first $0 \%$ stretch condition. Following the first $12 \%$ stretch cells were held at the second $0 \%$ stretch for $30 \mathrm{~min}$, to allow the actin cytoskeleton to stabilize (Krishnan et al., 2009), before traction force was analyzed. There was a dramatic decrease in traction force measured during the second $0 \%$ stretch condition, with traction force being significantly reduced compared to both the first $12 \%$ and $0 \%$ stretch conditions. Indicating that traction force is attenuated following a $12 \%$ increase in mechanical stretch. During the second $12 \%$ stretch traction forces increase significantly compared to the second $0 \%$ stretch condition (Fig. 2B). The significant percentage increase in traction force during the second 12\% stretch was $19 \%$ larger 
than that produced by the first $12 \%$ stretch (Fig. $2 \mathrm{C}$ ). These results show that cells still retain the ability to increase traction force following mechanical stretching with large strains even after traction force attenuation has occurred.

\subsection{Alterations in stress fibers occur during large strain mechanical stretching}

Dynamic alterations in the actin cytoskeleton were visualized by expressing GFP-actin in HUVECs (Fig. 3A \& B). We observed aberrations in the morphology of stress fibers with a potential loss of stress fiber integrity (defined by a decrease in the fluorescent intensity of GFP-actin in small regions along the stress fiber) during the initial $12 \%$ stretch, which persisted for the second 12\% stretch (white arrows, Fig. 3A \& B). Fluorescence intensities along the stress fibers were analyzed to evaluate the extent of stress fiber disruption. Fluorescence intensities of stretched stress fibers dropped significantly lower in certain regions compared to the consistent high intensities observed from stress fibers prior to stretching (Fig. 3C). We believe these drops in intensity correspond to the loss of stress fiber integrity induced by mechanical stretching. Although it has been indicated that the actin cytoskeleton can rupture or unfold during mechanical stretching (Gavara et al., 2008; Nekouzadeh et al., 2008), this is the first time, to our knowledge, that these disruptions to stress fiber integrity has been directly visualized. After the second round of $12 \%$ stretch no additional alterations in stress fiber structure were observed. With the loss of stress fiber integrity during stretch accompanied by post-stretch diminished traction forces, we infer that mechanical stretch results in a loss of stress fiber integrity resulting in traction force attenuation. Not all of the stress fibers where damaged by the first round of stretch as we were able to visualize intact stress fibers (Fig. 3A \& B). We propose that the stress fibers that retained their integrity following the initial stretch are responsible for the increase in traction force during the second round of stretch.
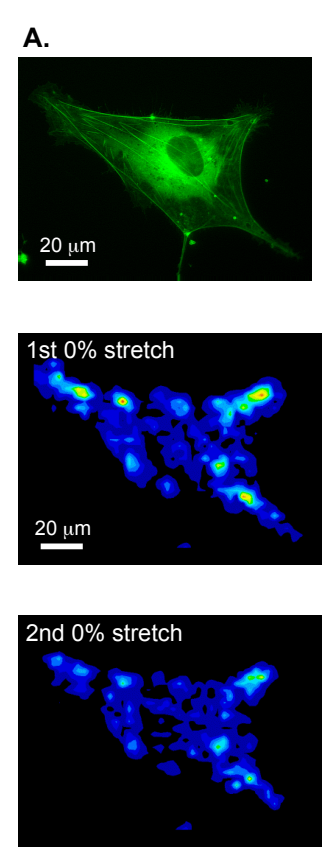
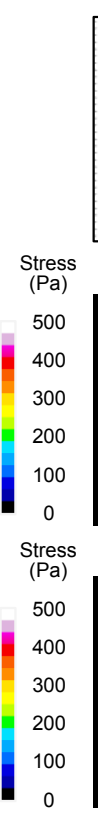
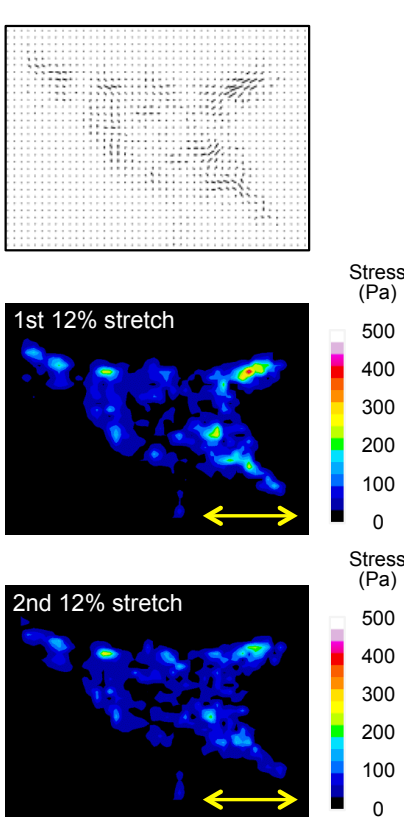

B.
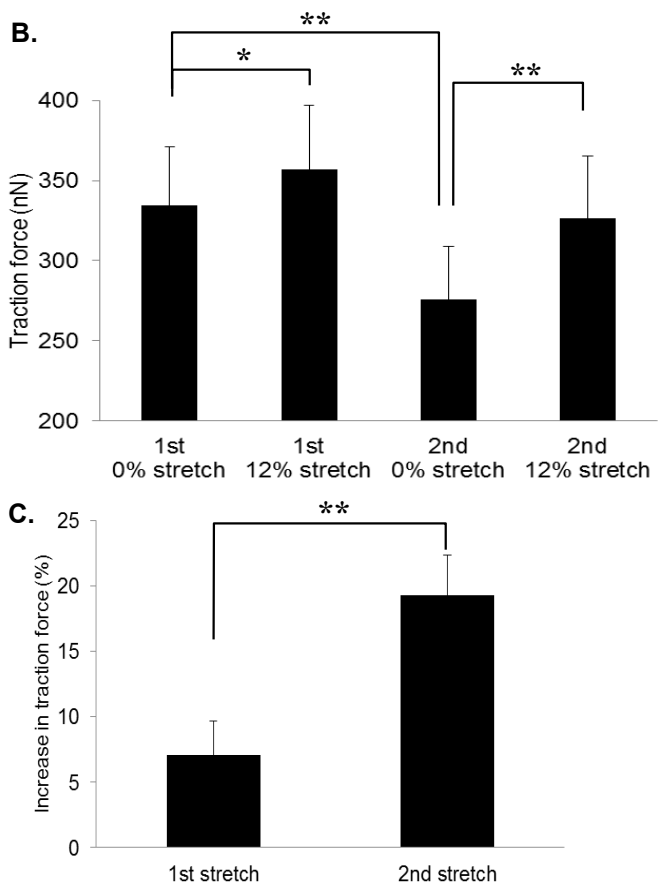

Fig. 2. Traction force increases following attenuation induced by mechanical stretch.

(A) Traction forces of a single cell, for each stage of the consecutive mechanical stretching and release cycle. Baseline traction forces prior to the first mechanical stretch (1st $0 \%$ stretch), traction forces during the first mechanical stretch (1st $12 \%$ stretch), traction forces following a $30 \mathrm{~min}$ rest prior to the second mechanical stretch (2nd $0 \%$ stretch), and traction forces during the second mechanical stretch (2nd 12\% stretch). Yellow arrows indicate direction of uniaxial stretch. A vector image indicates direction of traction forces. (B) Traction force analysis following consecutive mechanical stretching and release cycles: first $0 \%$ stretch, first $12 \%$ stretch, second $0 \%$ stretch and second $12 \%$ stretch. There was a slight but significant increase in traction force during the first $12 \%$ stretch $(\mathrm{p}=0.04, \mathrm{n}=13)$, this was followed by a significant attenuation of traction force during the second $0 \%$ stretch phase $(p=0.0004, n=13)$. A more significant increase in traction force was observed during the second $12 \%$ stretch $(\mathrm{p}=0.0002, \mathrm{n}=13)$. (C) Percentage increase in traction forces during mechanical stretching. Increases in traction force were significantly larger during the second $12 \%$ stretch than during the first $(\mathrm{p}=0.0002, \mathrm{n}=13)$. Data presented as mean \pm SEM. 


\section{Discussion}

In this study, traction forces in cells were measured during consecutive $12 \%$ mechanical stretching to investigate whether traction forces were able to increase once they become attenuated. We show for the first time, that traction forces are able to increase during a second round of mechanical stretching following attenuation. Alterations in the actin cytoskeleton have been reported to be involved in the attenuation of traction forces. With studies discussing, changes in actin polymerization, overstretching of stress fibers, rupturing and/or unfolding of actin filament cross-links and actomyosin cross-bridges as potential contributors to traction force attenuation (Costa et al., 2002; Gavara et al., 2008; Lim et al., 2012; Rosenblatt et al., 2007; Trepat et al., 2007; Trepat et al., 2006). However, no definitive evidence has been put forward to confirm these theories. In this study, we directly visualized stress fibers during mechanical stretching and observed a loss in stress fibers integrity accompanied by traction force attenuation. The strain of the mechanical stretching loaded onto cells in this study, $12 \%$ strain, was similar to strains used in previous studies reporting traction force attenuation (Gavara et al., 2008; Krishnan et al., 2009). However, studies investigating extracellular extracted stress fibers report breaks in stress fibers occur, due to shearing, when strains of 200-400\% are applied (Deguchi et al., 2006; Matsui et al., 2013). The reasons for this discrepancy are yet to be understood. Ablating stress fibers, using laser irradiation, causes redistribution of traction forces in single cells. Following the ablation of one stress fiber the pre-stressed cellular substrate relaxed enabling intact stress fibers to extend shifting the released tension from the ablated stress fiber to the remaining stress fibers (Kumar et al., 2006). In this study, we observed a loss of stress fiber integrity due to the potential shearing of stress fibers, which is very similar to stress fiber ablation. Thus, traction forces could be redistributed across intact stress fibers due to attenuation. The redistribution of traction forces during mechanical stretching could induce cellular remodeling, which has been reported during consecutive stretching (Hayakawa et al., 2001; Krishnan et al., 2012; Lee et al., 2010; Mitsuoka et al., 2012; Nagayama et al., 2012). Due to the redistribution of traction forces different intact stress fibers are now under strain, allowing for an increase in traction force to take place following another increase in mechanical stretch.

We report that cell traction force is able to increase in response to mechanical stretching even in conditions were traction force is attenuated. Our theory for how this is achieved is as follows... During the initial mechanical stretch, traction force in stress fibers increases. In some stress fibers, the traction force overwhelms their breaking point and stress fiber integrity is lost under the stress. This loss in stress fiber integrity could induce the attenuation in traction forces in the second $0 \%$ stretch condition. During the second mechanical stretch, the damaged stress fibers would no longer be involved in traction force increase. Instead, undamaged stress fibers maybe responsible for the increase in traction force. Although additional aberrations in stress fibers were difficult to find during the second mechanical stretch, it is not unreasonable to think further changes to the actin cytoskeleton could occur. For example the increase in traction force following the second $12 \%$ stretch is larger than that observed in the first $12 \%$ stretch, this difference could be due to cell stiffening after mechanical stimulations (Krishnan et al., 2012; Nagayama et al., 2012). In conclusion, traction force increases under mechanical stretching in cells even after traction forces have become attenuated due to anterior overstretching. Traction force attenuation is accompanied by stress fiber aberrations during mechanical stretching, which may result in a loss of stress fiber integrity. We proposed that it is the redistribution of tension to intact stress fibers, which accommodates the increase in traction force during consecutive mechanical stretching. 

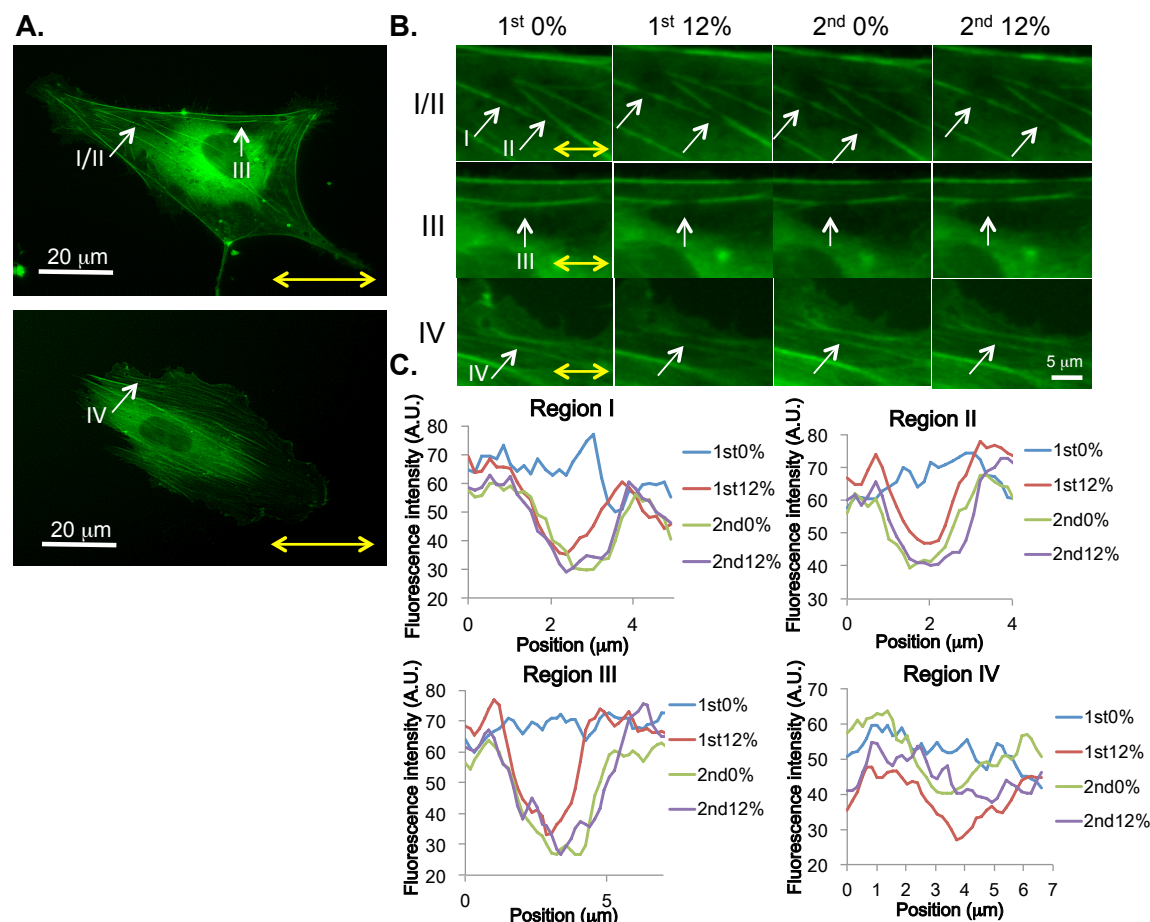

Fig. 3. Loss of stress fiber integrity observed during the initial $12 \%$ stretch.

(A) Cells in which loss of stress fiber integrity was observed. (B) Examples of regions with stress fiber aberrations (I-IV) over consecutive static and stretch conditions. Note that the stress fibers exhibit black voids, which maybe due to a loss of stress fibers integrity (white arrowheads). Yellow arrows indicate direction of uniaxial stretch. (C) Fluorescence intensity along highlighted stress fibers (indicated with marks "I-IV" in (A) and (B), respectively) show a decrease in intensity in these regions following the $1^{\text {st }} 12 \%$ stretch.

\section{Conflict of interest statement}

The authors declare that there are no conflicts of interest.

\section{Acknowledgments}

The authors are grateful to Dr. Masashige Shinkai for his kind advice and for gifting materials for the gels and Mr. Taro Watanabe for his technical assistance. This study was supported, in part, by a grant for the Translational Systems Biology and Medicine Initiative (TSBMI) from the Ministry of Education, Culture, Sports, Science, and Technology of Japan, and by Grants-in-Aid of JSPS KAKENHI Grant Number JP 15H01800, Grant Number JP 25820017, and Grant Number JP 15F15104.

\section{References}

Burton, K., Park, J.H., Taylor, D.L., Keratocytes generate traction forces in two phases, Molecular Biology of the Cell, Vol. 10, No. 11 (1999), pp.3745-3769.

Butler, J.P., Tolic-Norrelykke, I.M., Fabry, B., Fredberg, J.J., Traction fields, moments, and strain energy that cells exert on their surroundings, American journal of physiology. Cell physiology, Vol. 282, No. 3 (2002), pp.C595-605.

Costa, K.D., Hucker, W.J., Yin, F.C., Buckling of actin stress fibers: a new wrinkle in the cytoskeletal tapestry, Cell motility and the cytoskeleton, Vol. 52, No. 4 (2002), pp.266-274.

Decave, E., Garrivier, D., Brechet, Y., Fourcade, B., Bruckert, F., Shear flow-induced detachment kinetics of Dictyostelium discoideum cells from solid substrate, Biophysical journal, Vol. 82, No. 5 (2002), pp.2383-2395.

Deguchi, S., Ohashi, T., Sato, M., Tensile properties of single stress fibers isolated from cultured vascular smooth muscle cells, Journal of biomechanics, Vol. 39, No. 14 (2006), pp.2603-2610.

Gaudet, C., Marganski, W.A., Kim, S., Brown, C.T., Gunderia, V., Dembo, M., Wong, J.Y., Influence of type I collagen surface density on fibroblast spreading, motility, and contractility, Biophysical journal, Vol. 85, No. 5 (2003), pp.3329-3335.

Gavara, N., Roca-Cusachs, P., Sunyer, R., Farre, R., Navajas, D., Mapping cell-matrix stresses during stretch reveals inelastic 
reorganization of the cytoskeleton, Biophysical journal, Vol. 95, No. 1 (2008), pp.464-471.

Gavara, N., Sunyer, R., Roca-Cusachs, P., Farre, R., Rotger, M., Navajas, D., Thrombin-induced contraction in alveolar epithelial cells probed by traction microscopy, J Appl Physiol (1985), Vol. 101, No. 2 (2006), pp.512-520.

Hayakawa, K., Sato, N., Obinata, T., Dynamic reorientation of cultured cells and stress fibers under mechanical stress from periodic stretching, Experimental cell research, Vol. 268, No. 1 (2001), pp.104-114.

Krishnan, R., Canovic, E.P., Iordan, A.L., Rajendran, K., Manomohan, G., Pirentis, A.P., Smith, M.L., Butler, J.P., Fredberg, J.J., Stamenovic, D., Fluidization, resolidification, and reorientation of the endothelial cell in response to slow tidal stretches, American journal of physiology. Cell physiology, Vol. 303, No. 4 (2012), pp.C368-375.

Krishnan, R., Park, C.Y., Lin, Y.C., Mead, J., Jaspers, R.T., Trepat, X., Lenormand, G., Tambe, D., Smolensky, A.V., Knoll, A.H., Butler, J.P., Fredberg, J.J., Reinforcement versus fluidization in cytoskeletal mechanoresponsiveness, PloS one, Vol. 4, No. 5 (2009), pp.e5486.

Kumar, S., Maxwell, I.Z., Heisterkamp, A., Polte, T.R., Lele, T.P., Salanga, M., Mazur, E., Ingber, D.E., Viscoelastic retraction of single living stress fibers and its impact on cell shape, cytoskeletal organization, and extracellular matrix mechanics, Biophysical journal, Vol. 90, No. 10 (2006), pp.3762-3773.

Lam, R.H., Weng, S., Lu, W., Fu, J., Live-cell subcellular measurement of cell stiffness using a microengineered stretchable micropost array membrane, Integrative biology : quantitative biosciences from nano to macro, Vol. 4, No. 10 (2012), pp.1289-1298.

Lee, C.F., Haase, C., Deguchi, S., Kaunas, R., Cyclic stretch-induced stress fiber dynamics - dependence on strain rate, Rho-kinase and MLCK, Biochemical and biophysical research communications, Vol. 401, No. 3 (2010), pp.344-349.

Lim, S.M., Trzeciakowski, J.P., Sreenivasappa, H., Dangott, L.J., Trache, A., RhoA-induced cytoskeletal tension controls adaptive cellular remodeling to mechanical signaling, Integrative biology : quantitative biosciences from nano to macro, Vol. 4, No. 6 (2012), pp.615-627.

Markidou, A., Shih, W.Y., Shih, W.-H., Soft-materials elastic and shear moduli measurement using piezoelectric cantilevers, Review of Scientific Instruments Vol. 76, (2005), pp.064302.

Matsui, T.S., Sato, M., Deguchi, S., High extensibility of stress fibers revealed by in vitro micromanipulation with fluorescence imaging, Biochemical and biophysical research communications, Vol. 434, No. 3 (2013), pp.444-448.

Mitsuoka, Y., Tsukamoto, A., Iwayoshi, S., Furukawa, K.S., Ushida, T., High Time Resolution Time-Lapse Imaging Reveals Continuous Existence and Rotation of Stress Fibers under Cyclic Stretch in HUVEC, Journal of Biomechanical Science and Engineering, Vol. 7, No. 2 (2012), pp.188-198.

Munevar, S., Wang, Y.L., Dembo, M., Regulation of mechanical interactions between fibroblasts and the substratum by stretch-activated Ca2+ entry, Journal of cell science, Vol. 117, No. Pt 1 (2004), pp.85-92.

Nagayama, K., Adachi, A., Matsumoto, T., Heterogeneous response of traction force at focal adhesions of vascular smooth muscle cells subjected to macroscopic stretch on a micropillar substrate, Journal of biomechanics, Vol. 44, No. 15 (2011), pp.2699-2705.

Nagayama, K., Kimura, Y., Makino, N., Matsumoto, T., Strain waveform dependence of stress fiber reorientation in cyclically stretched osteoblastic cells: effects of viscoelastic compression of stress fibers, American journal of physiology. Cell physiology, Vol. 302, No. 10 (2012), pp.C1469-1478.

Nekouzadeh, A., Pryse, K.M., Elson, E.L., Genin, G.M., Stretch-activated force shedding, force recovery, and cytoskeletal remodeling in contractile fibroblasts, Journal of biomechanics, Vol. 41, No. 14 (2008), pp.2964-2971.

Pavan, T.Z., Madsen, E.L., Frank, G.R., Adilton, O.C.A., Hall, T.J., Nonlinear elastic behavior of phantom materials for elastography, Physics in medicine and biology, Vol. 55, No. 9 (2010), pp.2679-2692.

Rosenblatt, N., Hu, S., Suki, B., Wang, N., Stamenovic, D., Contributions of the active and passive components of the cytoskeletal prestress to stiffening of airway smooth muscle cells, Annals of biomedical engineering, Vol. 35, No. 2 (2007), pp.224-234.

Teixeira, A.I., Ilkhanizadeh, S., Wigenius, J.A., Duckworth, J.K., Inganas, O., Hermanson, O., The promotion of neuronal maturation on soft substrates, Biomaterials, Vol. 30, No. 27 (2009), pp.4567-4572.

Tolic-Norrelykke, I.M., Butler, J.P., Chen, J., Wang, N., Spatial and temporal traction response in human airway smooth muscle cells, American journal of physiology. Cell physiology, Vol. 283, No. 4 (2002), pp.C1254-1266.

Trepat, X., Deng, L., An, S.S., Navajas, D., Tschumperlin, D.J., Gerthoffer, W.T., Butler, J.P., Fredberg, J.J., Universal physical responses to stretch in the living cell, Nature, Vol. 447, No. 7144 (2007), pp.592-595.

Trepat, X., Puig, F., Gavara, N., Fredberg, J.J., Farre, R., Navajas, D., Effect of stretch on structural integrity and micromechanics of human alveolar epithelial cell monolayers exposed to thrombin, American journal of physiology. Lung cellular and molecular physiology, Vol. 290, No. 6 (2006), pp.L1104-1110.

Wang, J.H., Lin, J.S., Cell traction force and measurement methods, Biomechanics and modeling in mechanobiology, Vol. 6, No. 6 (2007), pp.361-371.

Yang, Z., Lin, J.S., Chen, J., Wang, J.H., Determining substrate displacement and cell traction fields--a new approach, Journal of theoretical biology, Vol. 242, No. 3 (2006), pp.607-616. 